III Sección: Educación, producción y finanzas

\title{
Un acercamiento a las finanzas de la Federación de Estudiantes de la Universidad de Costa Rica durante la década de 1960
}

\author{
Marco Garita Mondragón \\ Universidad de Costa Rica, Costa Rica \\ marco.garita@ucr.ac.cr \\ https://orcid.org/0000-0003-4821-8714
}

Recibido: 15 de setiembre de 2020

Aceptado: 2 de noviembre de 2020

Resumen: Mediante el análisis de los informes financieros de la Federación de Estudiantes de la Universidad de Costa Rica (FEUCR) a lo largo de la década de 1960, se demostrará que el contexto de expansión de las matrículas universitarias de ese decenio junto a importantes transformaciones en la estructura organizativa y económica de la federación permitieron una mayor participación del estudiantado en la opinión pública, al permitirles costear publicaciones periódicas, actividades de carácter ideológico como los congresos estudiantiles, publicaciones en prensa y proyectos de acción social, que sentarían las bases económicas para una mayor participación juvenil en la esfera pública.

Palabras clave: Movimiento estudiantil; Federación de Estudiantes de la Universidad de Costa Rica; finanzas del movimiento estudiantil; juventud; Costa Rica

\section{An Approach to the Finances of the University of Costa Rica's Federation of Students during the 1960s}

\section{(ब) $(\Theta \odot$}

La Revista Estudios es editada por la Universidad de Costa Rica y se distribuye bajo una Licencia Creative Commons Atribución-NoComercial-CompartirIgual 3.0 Costa Rica. Para más información envíe un mensaje a 
Abstract: Through the analysis of the financial reports of the University of Costa Rica's Federation of Students (FEUCR for its acronym in Spanish) throughout the 1960s, I will show that the context of expansion of university enrollments of that decade together with important transformations in organizational and economic structure of the Federation, allowed a greater participation of the students in public opinion, by allowing them to pay for periodical publications, ideological activities such as student congresses, press publications and social action projects, which would lay the economic foundations for a greater youth participation in the public sphere.

Keywords: Student movement; University of Costa Rica's Federation of Students; Student movement finances; Youth; Costa Rica

\section{Introducción}

El análisis de las juventudes ha tenido un auge en los últimos años en la academia costarricense, lo que ha repercutido en la publicación de importantes investigaciones sobre este tema (Molina Jiménez, 2019a; Molina Jiménez \& Díaz Arias, 2018). En ellas ha quedado clara la heterogeneidad de las juventudes y su relación con el contexto histórico en el que se desarrollan (Souto Kustrín, 2007). Esas investigaciones han enriquecido y desmitificado muchos supuestos sobre las juventudes costarricenses y se han sumado a un área antes dominada por las investigaciones clásicas de Óscar Arias Sánchez, Luis Barahona Jiménez, Paulino González Villalobos, Edelberto Torres-Rivas, y Carlos Picado Rojas (Arias Sánchez, 1970; Barahona Jiménez, 1972, 1976; González, 1985; González Villalobos, 1987; Picado Rojas, 1991; Torres-Rivas, Bronfenmajer, Cassá, Molina Chocano, \& James-Bryan, 1989).

La mayoría de esas investigaciones se han realizado desde enfoques que premian el análisis de las políticas públicas sobre la cuestión juvenil, el cambio cultural, la participación política de la juventud, sus características sociodemográficas, las memorias de los protagonistas de diversos movimientos sociales y la cuestión de la ciudadanía juvenil. Esa amplia gama de temas ha estado a tono con la historiografía regional más reciente (Acevedo Tarazona \& Samacá Alonso, 2011; Bonavena \& Millán, 2018).

\section{(c) (i) ()}

La Revista Estudios es editada por la Universidad de Costa Rica y se distribuye bajo una Licencia Creative Commons Atribución-NoComercial-CompartirIgual 3.0 Costa Rica. Para más información envíe un mensaje a 
A pesar de la diversidad de las investigaciones sobre el tema, existen pocos trabajos que profundicen en los elementos organizativos y administrativos del movimiento estudiantil centroamericano, específicamente sobre la cuestión del financiamiento de sus organizaciones. Los trabajos de Ricardo Argueta Hernández y Juan Antonio Gutiérrez Slon profundizan en lo primero, pero omiten por completo la cuestión del financiamiento (Argueta Hernández, 2004; Gutiérrez Slon, 2015). Existen muy pocos trabajos que se ocupen de este problema en la historiografía internacional de los movimientos estudiantiles. Gert van Maanen y Philip G. Altbach, por medio del análisis de diversas organizaciones internacionales de estudiantes durante la Guerra Fría, como la Unión Internacional de Estudiantes (UIE) y la Conferencia Internacional de Estudiantes (CIE), argumentan que uno de los principales elementos que limitaron la autonomía de estas "internacionales estudiantiles" fue su dependencia económica respecto a Estados, fondos universitarios, organizaciones políticas y/o religiosas. Hacia 1970 se estimaba que cerca del 90 por ciento de los recursos de estas organizaciones provenían de fondos no estudiantiles (Altbach, 1970a, pp. 51-61; Van Maanen, 1966). Como se verá en las siguientes páginas, las buenas relaciones construidas entre algunos sectores estudiantiles con las autoridades universitarias favorecieron para que la Universidad de Costa Rica financiara en parte la institucionalidad estudiantil, aunque a partir de la década de 1960, bajo un contexto de crecimiento de matrículas estudiantiles y burocratización del principal órgano estudiantil, la principal fuente de financiamiento variaría.

Es por ello que es objetivo de esta investigación realizar una primera aproximación al estudio de la estructura financiera de la Federación de Estudiantes Universitarios de Costa Rica (FEUCR) durante la década de 1960. Específicamente, se abordará el análisis de las finanzas de la Federación por medio del estudio de los balances y reportes financieros elaborados por la Secretaría de Finanzas de dicha organización, como también de los presupuestos realizados. Interesa acercarse a la estructura de ingresos y egresos para conocer las prioridades 
financieras de la organización. Esa documentación se encuentra disponible en el Archivo Universitario Rafael Obregón Loría (AUROL) en el fondo FEUCR. ${ }^{\text {' Se }}$ constatará que las finanzas de la organización estudiantil se fortalecieron gracias a la creación de un impuesto estudiantil lo cual, en un contexto de crecimiento de las matrículas en la Universidad de Costa Rica y de renovación organizativa del movimiento estudiantil universitario, le permitió a la federación complejizar su estructura administrativa y financiar su participación en la opinión pública nacional e internacional.

En un primer apartado se analizará el crecimiento de las matrículas universitarias costarricenses durante la década de 1960 y su relación con el radicalismo juvenil de esa época. En el segundo apartado, se estudiará el financiamiento del principal órgano estudiantil de la Universidad de Costa Rica, con el objeto de demostrar que hacia inicios de la década en cuestión, la estructura económica de la Federación estudiantil tendió a consolidarse gracias a importantes cambios en su estructura de ingresos. En el tercer apartado, por medio de un presupuesto realizado en 1968, se profundizará en el gasto de la organización con el objetivo de estudiar la estructura de egresos. Finalmente, se incluirá una sección de conclusiones, donde se recapitularán los principales hallazgos de investigación.

\section{Crecimiento de las matrículas y radicalismo político}

La década de 1960 en Costa Rica es sinónimo de inversión y expansión educativa, aunque sería la década de 1970 la que vería los mejores índices en ambos rubros. No obstante, el lento crecimiento que ambas materias desempeñaron desde la década de 1950 se aceleraría especialmente a mediados de los 60’s (Molina Jiménez, 2016, pp. 351, 355, 2018). De hecho, como se observa en el Gráfico 1, en esa década la expansión de las matrículas en la Universidad de Costa Rica se triplicó. En 1960, la institución le abrió sus puertas a 3.801 estudiantes, en 1965 a 5.762 y en 1970 a 12.913. Ese crecimiento se debió en parte al incremento que experimentó la educación secundaria en la década de 1950, el

\section{(c) (i) (2)}

La Revista Estudios es editada por la Universidad de Costa Rica y se distribuye bajo una Licencia Creative Commons Atribución-NoComercial-CompartirIgual 3.0 Costa Rica. Para más información envíe un mensaje a 
aumento de la inversión estatal en infraestructura y equipo educativo, la diversificación de las carreras universitarias y el énfasis que le dio la institución a la docencia tras la reforma de 1957 que culminó con la creación de la Escuela de Estudios Generales (Molina Jiménez, 2016, pp. 394-395). No obstante, existieron importantes conflictos que involucraron a la UCR, el Estado, los estudiantes y padres de familia en relación al presupuesto de esta primera y a las políticas institucionales de acceso a la universidad (Molina Jiménez, 2016, pp. 348-349, 2019b, 2020).

A pesar de ese incremento en términos absolutos de las matrículas estudiantiles, persistieron algunas brechas respecto al acceso. Las mujeres representaron en promedio apenas un 35,5 por ciento de las matrículas universitarias durante el periodo 1953-1970. Mientras que, por otro lado, la matrícula estudiantil se concentró mayoritariamente en hijos de las clases medias costarricenses: entre 1967 y 1973, únicamente el 2,5 por ciento de los 18.257 padres de familias de estudiantes pertenecían a los sectores laborales menos calificados (Molina Jiménez, 2016, pp. 402-405). Lo anterior repercutió en que la radicalización estudiantil hacia finales de la década fuera principalmente llevada a cabo por estudiantes pertenecientes a los estratos medios del país (Arias Sánchez, 1970, pp. 41-51).

\section{(c) (i)(2)}

La Revista Estudios es editada por la Universidad de Costa Rica y se distribuye bajo una Licencia Creative Commons Atribución-NoComercial-CompartirIgual 3.0 Costa Rica. Para más información envíe un mensaje a 


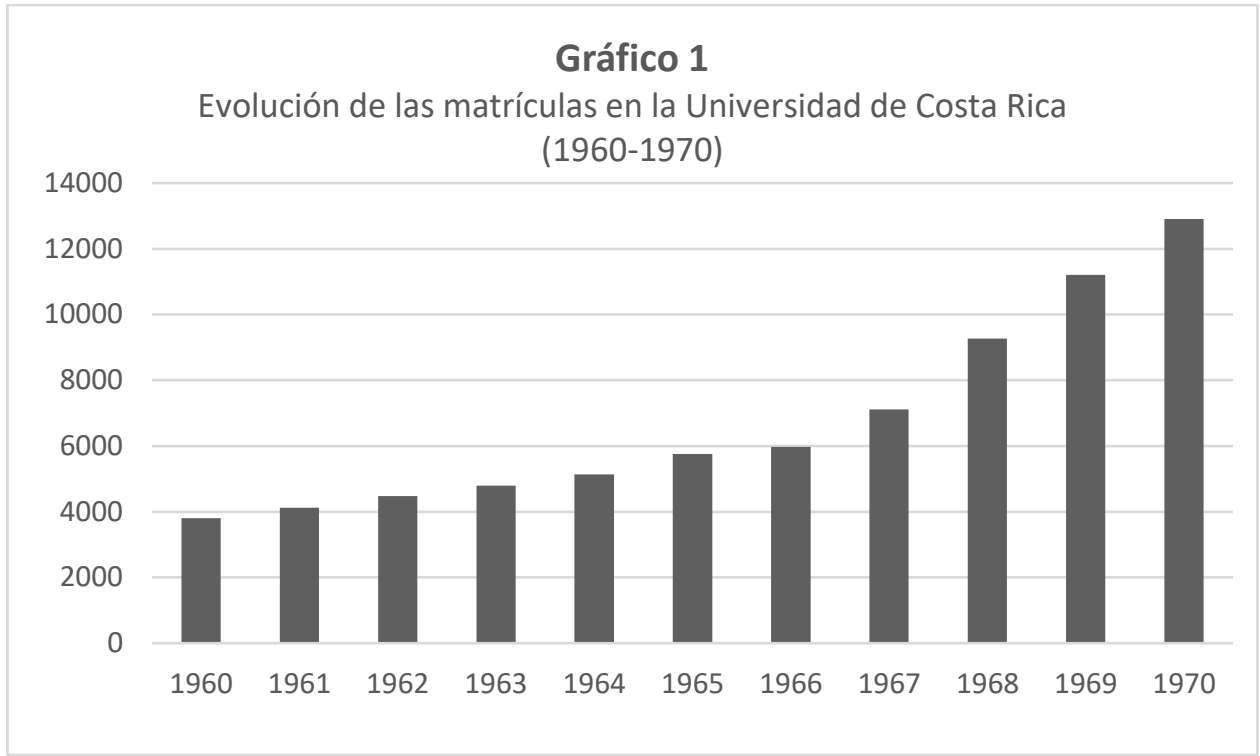

Fuente: elaboración propia con datos de Molina, 2017:

De acuerdo a Eric Hobsbawm, durante la segunda posguerra se configuró una nueva dinámica juvenil caracterizada por su idealización, la creación de un mercado de consumo dirigido a los jóvenes-consumidores y por su profundo internacionalismo (Hobsbawm, 1999, pp. 327-331). La expansión de las matrículas educativas, especialmente en el mundo urbano, favoreció la extensión de la edad de dependencia de los jóvenes respecto a sus padres. Muchos de ellos terminarán influenciados por la Revolución Cubana, los procesos de descolonización en África, la Guerra de Vietnam y la revolución sexual, generando un mayor activismo político que desencadenará en la "primera rebelión global" de 1968 (Klimke \& Nolan, 2018, p. 1). En Centroamérica, la preocupación de la Iglesia Católica por la juventud, especialmente marcada a partir del Concilio Vaticano II (1962-1965), jugaría un rol fundamental en esa radicalización (Chaves Zamora, 2020; Chávez, 2014).

Costa Rica no escapó de ese proceso de expansión educativa y de radicalismo juvenil. Sin embargo, a diferencia de muchos estudiantes latinoamericanos que optaron por engrosar las filas de diversos movimientos guerrilleros rurales y urbanos (Kruijt, 2009; Pozzi \& Pérez, 2012), el estudiantado costarricense optó por mesura y prefirió el uso de mecanismos institucionales y

\section{(c) (i) (-)}

La Revista Estudios es editada por la Universidad de Costa Rica y se distribuye bajo una Licencia Creative Commons Atribución-NoComercial-CompartirIgual 3.0 Costa Rica. Para más información envíe un mensaje a revistaestudios.eeg@ucr.ac.cr. 
nacionalistas para su activismo político, muchas veces emulando la institucionalidad costarricense. Según Randall Chaves Zamora, la cultura política del estudiantado universitario de la década de 1960 se incorporó "a las dinámicas políticas de su entorno académico mediante acciones circunscritas a la cultura institucional de su alma mater" lo que los llevó a desarrollar acciones políticas mesuradas en busca de acercarse a las tradiciones institucionales de la Universidad y el Estado costarricense, con el afán de distanciarse de elementos discursivos que los relacionase con las izquierdas nacionales e internacionales. Para el mismo autor, las protestas callejeras contra en contrato-ley entre el Estado y la Aluminum Company of America (ALCOA) en 1970 significó una ruptura a esa dinámica porque los estudiantes se movilizaron masivamente por razones no estrictamente universitarias y desarrollaron nuevas formas de protesta aunque sus acciones estuvieron mediadas por tradiciones del movimiento social del país, como su nacionalismo (Chaves Zamora, 2018a, 2018b).

Durante la década, el Estado se preocupará más a fondo sobre la cuestión juvenil al fomentar el desarrollo de políticas públicas orientadas a redimir por vías institucionales las demandas juveniles, entre las que destacaron la creación del Movimiento Nacional de Juventudes en 1965, la creación del Ministerio de Cultura, Juventud y Deportes en 1971 y la ampliación de los derechos políticos-electorales al extenderse el derecho al voto a los mayores de 18 años (previamente la edad mínima era de 21 años) en ese mismo año (M. Salazar Montes, 2018; M. F. Salazar Montes, 2013).

\section{Un acercamiento a las finanzas de la FEUCR}

Desde la fundación en agosto de 1941 de la Unión Nacional de Estudiantes de Costa Rica (UNECR) y el Consejo Estudiantil Universitario, la organización oficial estudiantil de la Universidad de Costa Rica sufrió importantes transformaciones organizativas. En 1953 gracias a una consulta general a los miembros de la UNECR, se decidió la reorganización del movimiento universitario a favor de la creación de una federación de estudiantes, lo que repercutió en la creación de diferentes

La Revista Estudios es editada por la Universidad de Costa Rica y se distribuye bajo una Licencia Creative Commons Atribución-NoComercial-CompartirIgual 3.0 Costa Rica. Para más información envíe un mensaje a 
Revista Estudios, (41), 2020.

Diciembre 2020-Mayo 2021

ISSN 1659-3316

Garita Mondragón Marco

asociaciones estudiantiles entre 1953 y 1956, que sentaron las bases para la fundación en 1959 de la Federación de Estudiantes Universitarios de Costa Rica (FEUCR) ("Historia de la FEUCR”, 1967).

No hay certeza de cómo operó el financiamiento estudiantil a lo largo de la década de 1940. No obstante, en 1954 la universidad decidió brindarle una subvención económica al Consejo Estudiantil Universitario. De acuerdo al rector de la institución en ese momento, Rodrigo Facio Brenes (1917-1961), el subsidio era un reconocimiento a la seriedad y buenas relaciones que tenían los líderes estudiantiles con las autoridades universitarias. De acuerdo al intelectual, "la idea es ir liberando al organismo estudiantil de la necesidad de imprimirles carácter lucrativo a sus festividades para fines de su propia financiación" (Universidad de Costa Rica, 1955, p. 9). En ese momento el auxilio económico ascendió a los $\mathbb{} 700$, en 1956 se actualizó el monto a $\mathbb{1} 1.500$ y en 1957 se duplicó el subsidio para llegar a los $\mathbb{} \$ 3.600$ (Universidad de Costa Rica, 1955, p. 515, 1957, p. 14, 1958, p. 743). No obstante, esa última cifra no se volvería a actualizar a lo largo de la década de 1960, ocasionando que en términos reales el subsidio tendiera hacia la baja producto de la inflación.

Las buenas relaciones construidas por un sector del estudiantado y las autoridades universitarias se explican por el impacto que tuvo la Guerra Civil de 1948 en la universidad y por la dinámica política amparada en la Guerra Fría. Facio fue el principal líder del Centro para el Estudio de los Problemas Nacionales (CEPN) y uno de los principales intelectuales detrás del proyecto de modernización llevado a cabo por el Partido Liberación Nacional. Como funcionario universitario, se metió de lleno en la polarización política que afectó a la UCR en los albores de 1948, incluso llegando a ser donante, junto al Consejo Estudiantil Universitario, del Ejército de Liberación Nacional (Fumero, 1997). Tenían en común su asiduo anticomunismo, sus ansias por modernizar Costa Rica por medio de un proyecto de sustitución de importaciones y su rechazo a las dictaduras latinoamericanas. Eso los llevó a ser exponentes de un anticomunismo con características reformistas

La Revista Estudios es editada por la Universidad de Costa Rica y se distribuye bajo una Licencia Creative Commons Atribución-NoComercial-CompartirIgual 3.0 Costa Rica. Para más información envíe un mensaje a revistaestudios.eeg@ucr.ac.cr. 
(Molina Jiménez, 2009; Molina Jiménez \& Díaz Arias, 2017). Esas simpatías ideológicas entre una parte del estudiantado y la cúpula universitaria se trasladaron a lo largo de la década de 1950, como lo evidencia las mismas declaraciones de Facio y la nula participación de la oficialidad estudiantil en acciones que cuestionaran el orden universitario. De hecho, la única huelga universitaria de envergadura que se tiene constancia en la década, sucedió en 1954, cuando los universitarios se solidarizaron con las estudiantes del Colegio Superior de Señoritas y del Colegio Sion, ante los intentos de la Iglesia Católica y parte del gobierno de controlar sus formas de vestir y sus actividades deportivas (González Ortega, 2005, pp. 83-87). De acuerdo a Alfonso González Ortega, el decenio de 1950 estuvo caracterizado por un autoritarismo rígido y punitivista que pretendió alejar a las juventudes de la "contaminación comunista y somocista", lo que llevó al rechazo de las voces juveniles que se alejaban de ese ideal, a la vez que se reconocían a los jóvenes que coincidían con las luchas políticas de los adultos (González Ortega, 2005, pp. 1-51).

Hacia inicios de la década de 1960 el Consejo Superior de la recién fundada FEUCR, se encontraba en una situación financiera inestable, debido a que dependían de los ingresos que pudieran generar a raíz de la organización de diversas actividades culturales, como la Semana Universitaria, y al subsidio que le brindaba la Universidad de Costa Rica. Como vimos, ese último monto dejó de actualizarse desde 1957, presionando al estudiantado a buscar otras formas de financiamiento.

Ante esa situación, los miembros del Consejo se dieron a la tarea de proponer diversas alternativas a las autoridades universitarias. Una de ellas fue la propuesta de creación de un timbre o impuesto estudiantil y la otra fue la solicitud de un aumento del subsidio dado por la universidad. En la sesión 37 del Consejo Superior Estudiantil celebrada el 7 de enero de 1960 se acordó impulsar la creación del timbre por un monto de $\mathbb{\$ 5 , 0 0}$ cobrable a todo estudiante que matriculara una o más materias en la Universidad. Según el acuerdo, el cobro se llevaría a cabo una vez 
al año." El Consejo Universitario (CU) acordó que acogería ambas propuestas siempre y cuando la FEUCR presentara un informe de su movimiento financiero en 1959 y un proyecto de egresos para 1960.iii Es probable que los miembros del Consejo Superior no pudieran cumplir con los requerimientos del CU ya que no se tiene constancia de algún tipo de informe financiero sino hasta diciembre de 1961. iv De hecho, es a partir de 1962 que en los diferentes informes y presupuestos de la FEUCR se contempla el rubro del timbre estudiantil, aunque con modificaciones a la propuesta inicial, ya que al final se redujo el monto de los $\mathbb{C} 5,00$ propuestos inicialmente a $\mathbb{\mathbb { }} 4,00$, como se constata en la documentación financiera. ${ }^{\vee}$

No se tiene certeza de cuáles fueron las razones argumentadas por el CU para denegar el aumento propuesto por el estudiantado para aumentar el subsidio estudiantil. Tampoco se sabe por qué el subsidio no sufrió ninguna actualización a lo largo de la década de 1960 y se mantuvo estático en los $\mathbb{} \$ 3.600$ anuales. Es muy probable que el estancamiento del financiamiento público dado a la UCR durante la primera mitad del decenio (Molina Jiménez, 2016, p. 351) motivara a las autoridades universitarias a no aumentar ese monto y a los estudiantes a proponer medidas alternativas para financiar a la organización estudiantil.

En la Tabla 1 se puede observar la evolución de los ingresos de la FEUCR por el concepto del timbre estudiantil entre 1962 y 1970. Es necesario mencionar que los datos presentados para algunos años estuvieron incompletos debido a que muchas veces los informes financieros eran elaborados antes de la recolección total de los ingresos del timbre, lo que indica que este se pagaba en tractos. Además, en algunos años se tuvieron que estimar los ingresos ante la falta de información financiera relevante en las cajas y libros consultados. Para ello se procedió a multiplicar el total de matrículas del año por el monto del timbre, bajo el supuesto que esos fondos terminaron ingresando a las arcas estudiantiles. Realizadas esas aclaraciones, un primer elemento que se desprende de la tabla estudiada es el crecimiento constante a lo largo de la década de los ingresos por este rubro, en total sintonía con el crecimiento de las matrículas expuestas en el Gráfico 1.

La Revista Estudios es editada por la Universidad de Costa Rica y se distribuye bajo una Licencia Creative Commons Atribución-NoComercial-CompartirIgual 3.0 Costa Rica. Para más información envíe un mensaje a 
Revista Estudios, (41), 2020.

Diciembre 2020-Mayo 2021

ISSN 1659-3316

Garita Mondragón Marco

Se constata que entre 1962 y 1966 se dio un crecimiento lento de los ingresos

recolectados reales, pasando de $\$ 3.514,81$ a $\$ 3.612,97$ (o un máximo de $\$ 4.317,50$ si se basa en la estimación con los datos de matrículas), representando un crecimiento de 9,32 por ciento (o un máximo de 24,12 por ciento tomando como referencia la estimación). Sin embargo, a partir de 1967 los ingresos se disparan y llegan a su máximo en 1970 cuando la FEUCR recibió por este concepto \$15.849,06, es decir, más de un 300 por ciento de los ingresos de 1962.

Tabla 1

Evolución de ingresos FEUCR por concepto de impuesto estudiantil $(1962-1970)^{*}$

\begin{tabular}{l|l|l|l|l|l}
\hline Año & $\begin{array}{l}\text { Colones } \\
\text { corrientes** }\end{array}$ & Matrícula & $\begin{array}{l}\text { Tipo } \\
\text { cambio }\end{array}$ & $\begin{array}{l}\text { Dólares } \\
\text { corrientes }\end{array}$ & $\begin{array}{l}\text { Dólares de } \\
1970\end{array}$ \\
\hline 1962 & $18.136,00$ & 4479 & 6,625 & $2.737,51$ & $3.514,81$ \\
1963 & $19.168,00$ & 4792 & 6,625 & $2.893,28$ & $3.670,76$ \\
1964 & $18.988,00$ & 5133 & 6,625 & $2.866,11$ & $3.589,62$ \\
1965 & $23.048,00$ & 5762 & 6,625 & $3.478,94$ & $4.284,32$ \\
1966 & $20.000,00$ & 5975 & 6,625 & $3.018,87$ & $3.612,97$ \\
1967 & $42.928,00$ & 7109 & 6,625 & $6.479,70$ & $7.536,32$ \\
1968 & $59.000,00$ & 9265 & 6,625 & $8.905,66$ & $9.940,37$ \\
1969 & $44.848,00$ & 11212 & 6,625 & $6.769,51$ & $7.168,91$ \\
1970 & $105.000,00$ & 12913 & 6,625 & $15.849,06$ & $15.849,06$ \\
\hline
\end{tabular}

Fuente: Balance de prueba (al 28/09/1962); Informe de Secretaría de Finanzas (mayojunio 1964); Secretaría de Finanzas (08/08/1966); Estado de ingresos y egresos (al 30/06/1967); Estado de ingresos y egresos (al 31/05/1968); Molina, 2017: 8, 38.

*Los años de 1963, 1965 y 1969 se basan en estimaciones realizadas a partir de los datos de matrículas. En 1966 la FEUCR presupuestó $\mathbb{\$} 20.000$ por este concepto. Durante el segundo semestre de ese año se recolectaron $\mathbb{4} 4.436$. En 1968 la FEUCR presupuestó $\mathbb{L} 62.000$ pero al 31 de mayo solo se habían recaudado $\mathbb{W} 59.000$. En 1970

La expansión universitaria que se desarrolló en la década de 1960 no solo ocasionó que las matrículas se multiplicaran y se diera un proceso de regionalización de la educación superior con la creación en 1968 de la Sede de Occidente de la Universidad de Costa Rica (Fonseca Zúñiga, 2020), sino que también ocasionó que las finanzas estudiantiles se fortalecieran gracias a este timbre estudiantil. Incluso es probable que hacia finales de la década el impuesto

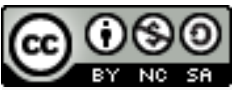

La Revista Estudios es editada por la Universidad de Costa Rica y se distribuye bajo una Licencia Creative Commons Atribución-NoComercial-CompartirIgual 3.0 Costa Rica. Para más información envíe un mensaje a 
aumentara de $\mathbb{4} 4,00$ a $\mathbb{\&} 8,00$, ya que el monto presupuestado y la cantidad de estudiantes matriculados para 1970 se acercan más a esta segunda realidad. Ese aumento del 100 por ciento del impuesto coincide con la reorganización del método de elección de los dirigentes de la Federación al establecerse el voto directo en los comicios generales de 1969 (Gutiérrez, 1969, p. 392; Molina Jiménez, 2016, p. 399), lo que podría sugerir que el aumento del timbre se pudo dar en parte para hacer frente al costo de la organización y financiamiento de las campañas electorales. El reglamento electoral de 1970 emitido por el Tribunal Universitario de Elecciones Estudiantiles para regular los comicios, establecía que esa entidad "designará una partida de su presupuesto para financiar la propaganda de los candidatos". vi

En la Tabla 2 se observa la evolución de los ingresos y pérdidas originadas en torno a la organización de la Semana Universitaria entre 1961 y 1969. Se observa que durante los años estudiados la organización de tal actividad siempre significó ganancias para la FEUCR. A pesar de que existieron algunos años en los cuales no se pudo encontrar información financiera de calidad para completar la tabla, sí se puede observar una clara tendencia hacia la baja. Es decir, conforme avanza la década de 1960 las ganancias generadas por esta actividad tienden a reducirse, con la notable excepción de 1966. En 1961 las actividades culturales y de ocio generaron ingresos reales por \$1.340,99 mientras que para 1969 las actividades le generaron a la Federación tan solo $\$ 327,69$, es decir, una reducción de más del 300 por ciento.

Es probable que dos procesos diferentes expliquen este fenómeno. Por un lado, la incertidumbre financiera de la FEUCR se redujo drásticamente gracias a los ingresos provenientes del timbre estudiantil lo que pudo haber reducido las ansias de lucro en la organización de las actividades de esta Semana para hacer frente a los gastos varios de la organización, como lo planteaba Rodrigo Facio en su informe de 1954. Por otro lado, es probable que los miembros del Consejo Superior cada vez más permitieran que las diferentes asociaciones estudiantiles organizaran sus propias actividades (bailes, especialmente) -con su respectivo derecho de cantina- 
Revista Estudios, (41), 2020.

Diciembre 2020-Mayo 2021

ISSN 1659-3316

Garita Mondragón Marco

haciendo que una parte de las ganancias obtenidas por las actividades, terminaran en las tesorerías de las respectivas asociaciones. Existen varios documentos de 1970 donde se constatan los ingresos a las arcas de la FEUCR a raíz del pago de insumos para la celebración de la Semana Universitaria de diversas asociaciones. vii Eso sugiere que el Consejo Superior optó por dejar de hacerse cargo de la organización directa de estas actividades aunque mantuvo cierto control financiero al convertirse en el proveedor de vasos, cervezas, licores y cigarrillos de las actividades organizadas por las asociaciones estudiantiles.

Tabla 2

Evolución de ganancias y pérdidas por Semana Universitaria (1961-1969)

\begin{tabular}{lllll}
\hline Año & $\begin{array}{l}\text { Colones } \\
\text { corrientes }\end{array}$ & $\begin{array}{l}\text { Tipo de } \\
\text { cambio }\end{array}$ & $\begin{array}{l}\text { Dólares } \\
\text { corrientes }\end{array}$ & $\begin{array}{l}\text { Dólares de } \\
\mathbf{1 9 7 0}\end{array}$ \\
\hline 1961 & $5.795,50$ & 5,61 & $1.033,07$ & $1.340,99$ \\
1962 & $3.773,10$ & 6,625 & 569,52 & 731,24 \\
1963 & & 6,625 & & \\
1964 & $3.787,30$ & 6,625 & 571,67 & 715,98 \\
1965 & & 6,625 & & \\
1966 & $5.226,50$ & 6,625 & 788,91 & 944,16 \\
1967 & & 6,625 & & \\
1968 & $3.056,05$ & 6,625 & 461,29 & 514,89 \\
1969 & $2.050,00$ & 6,625 & 309,43 & 327,69 \\
& & & & \\
\hline
\end{tabular}

Fuente: Balance por sumas (al 31/1//1961); Informe de ingresos y egresos de la Semana Universitaria (1962); Informe Semana Universitaria (1964); Secretaría de Finanzas (08/08/1966); Informe financiero Semana Universitaria (1968); Sesión ordinaria del Directorio (29/08/1969); Molina, 2017: 8.

Estas dos fuentes de financiamiento fueron las más importantes para la FEUCR durante la década de 1960. Aunque no fueron las únicas, llegaron a representar un gran porcentaje de los ingresos totales de la Federación. La falta de información para todos los años estudiados no permite construir unas adecuadas series sobre la distribución total del gasto y los ingresos totales de la Federación. Sin embargo, se intentará hacer una aproximación de ambos rubros a partir de los

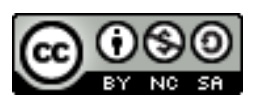

La Revista Estudios es editada por la Universidad de Costa Rica y se distribuye bajo una Licencia Creative Commons Atribución-NoComercial-CompartirIgual 3.0 Costa Rica. Para más información envíe un mensaje a revistaestudios.eeg@ucr.ac.cr. 
presupuestos de 1968. Aunque es importante destacar que a lo largo de la década existe un proceso de burocratización de la FEUCR, por lo que sus cuentas hacia esos años tendieron a complejizarse en comparación con los años iniciales. Para facilitar el análisis, de nuevo se redujeron las categorías con el objetivo de aglutinar diferentes rubros con gran similitud.

\section{Analizando el gasto: el presupuesto de 1968}

Para 1968, la Secretaría de Finanzas presupuestó ingresos por \$13.504,59 reales. Más del 77 por ciento de esos ingresos serían cubiertos por el timbre estudiantil, mientras que un 18,7 por ciento sería cubierto por la Universidad de Costa Rica por medio de diferentes subvenciones (incluyendo la subvención normal, la subvención al periódico estudiantil y un aporte extraordinario para la compra de mobiliario), un 3,7 por ciento de los ingresos serían generados a partir de la Semana Universitaria y el alquiler del Centro de Recreación, mientras que el restante 0,2 por ciento sería producto de las ganancias generadas por una compra de activos financieros que la FEUCR habría efectuado. Como se constata, hacia finales de la década las principales fuentes de ingreso estudiantil fueron los aportes por medio del timbre y las subvenciones universitarias. Aunque es notable que existió un impulso por diversificar los ingresos al incluirse la práctica de invertir dinero en acciones (es en 1967 la primera vez que aparece la cuenta de "inversiones transitorias" en los estados financieros).

\section{(c) (i) (2)}

La Revista Estudios es editada por la Universidad de Costa Rica y se distribuye bajo una Licencia Creative Commons Atribución-NoComercial-CompartirIgual 3.0 Costa Rica. Para más información envíe un mensaje a 
Tabla 3

Ingresos y egresos presupuestados por la FEUCR

\begin{tabular}{|c|c|c|c|c|}
\hline Ingresos & $\begin{array}{l}\text { Colones } \\
\text { corrientes }\end{array}$ & $\%$ & $\begin{array}{l}\text { Dólares } \\
\text { corrientes }\end{array}$ & $\begin{array}{l}\text { Dólares de } \\
1970\end{array}$ \\
\hline Cuota estudiantil & $62.000,00$ & 77,4 & $9.358,49$ & $10.445,82$ \\
\hline $\begin{array}{l}\text { Subvenciones } \\
\text { Ingresos }\end{array}$ & $15.005,00$ & 18,7 & $2.264,91$ & $2.528,06$ \\
\hline $\begin{array}{l}\text { generados } \\
\text { Ingresos por }\end{array}$ & $3.000,00$ & 3,7 & 452,83 & 505,44 \\
\hline inversiones & 150,00 & $\begin{array}{l}0,2 \\
100\end{array}$ & 22,64 & 25,27 \\
\hline $\begin{array}{l}\text { Total } \\
\text { Egresos }\end{array}$ & $80.155,00$ & 0 & $12.098,87$ & $13.504,59$ \\
\hline Salarios & $18.165,00$ & 22,7 & $2.741,89$ & $3.060,46$ \\
\hline Viáticos & $6.000,00$ & 7,5 & 905,66 & $1.010,89$ \\
\hline $\begin{array}{l}\text { Comisiones de } \\
\text { trabajo }\end{array}$ & $12.500,00$ & 15,6 & $1.886,79$ & $2.106,01$ \\
\hline Periódico & $15.000,00$ & 18,7 & $2.264,15$ & $2.527,21$ \\
\hline Gastos operativos & $12.490,00$ & 15,6 & $1.885,28$ & $2.104,33$ \\
\hline II CEU & $2.000,00$ & 2,5 & 301,89 & 336,96 \\
\hline $\begin{array}{l}\text { Publicaciones } \\
\text { Equipo para }\end{array}$ & $10.000,00$ & 12,5 & $1.509,43$ & $1.684,81$ \\
\hline deportes & $4.000,00$ & $\begin{array}{l}5,0 \\
100,\end{array}$ & 603,77 & 673,92 \\
\hline Total & $80.155,00$ & 0 & $12.098,87$ & $13.504,59$ \\
\hline
\end{tabular}

Fuente: Estado de ingresos y egresos al 31 de mayo de 1968.

En relación a los egresos es notable observar la gran cantidad de recursos utilizados por el concepto de salarios, lo que demuestra que hacia finales de la década la estructura interna de la FEUCR tendió hacia la burocratización. Del total presupuestado, un 22,7 por ciento estaba destinado al pago de salarios, horas extras, cotizaciones a la Caja Costarricense del Seguro Social y el pago de aguinaldos. Ese rubro incluía el salario del presidente, vicepresidente, secretario y guarda de seguridad. En una boleta de inscripción patronal fechada el 2 de enero 
de 1968 se establecía que el guarda de seguridad recibía un salario de aproximadamente $\$ 76$ reales mensuales y el secretario recibía la suma aproximada de $\$ 51$ reales al mes. viii El segundo egreso en importancia estuvo relacionado con la publicación de "El Universitario", el órgano oficial de la Federación, ya que se presupuestó un 18,7 por ciento del total. Entre los gastos operativos y de las diferentes comisiones se presupuestó más del 30 por ciento de los recursos, mientras que para el financiamiento de las publicaciones (como boletines informativos, publicaciones en prensa, etc.) se proyectó gastar un 12,5 por ciento de los ingresos totales.

De esa forma se puede observar que gran parte de los gastos de la Federación estaban orientados a hacerle frente a la administración interna de la organización y a su proyección estudiantil, tanto en el escenario nacional como internacional, por medio de los gastos en las diferentes comisiones y por la publicación de diversos textos dirigidos a la población estudiantil y nacional, lo que incluían el pago de campos pagados y cuñas radiales. Los datos fragmentarios que contamos para estudiar la evolución del gasto realizado por la FEUCR por concepto de "relaciones internacionales" evidencian una tendencia hacia la baja durante la década. Sin embargo es importante tener cuidado con la información presentada en los informes.

En los años de 1961, 1962 y 1964 la documentación financiera no distingue si estos gastos incluyen las cuotas para las organizaciones estudiantiles internacionales, los gastos de la comisión de relaciones internacionales y los costos de los cables y telegramas. En 1968 el presupuesto hace la distinción entre los gastos de la comisión mencionada y los gastos relacionados a la correspondencia, aunque en la Tabla 4 solo se incluye el presupuesto para la Comisión de Relaciones Internacionales. Es importante contrastar esta información con la correspondencia que recibió y envió la Federación a diferentes instancias u organizaciones internacionales, debido que a principios de la década de 1960 la correspondencia internacional es de gran tamaño. Esto ya lo había observado Luis Barahona 
Revista Estudios, (41), 2020.

Diciembre 2020-Mayo 2021

ISSN 1659-3316

Garita Mondragón Marco

Jiménez en la década de 1970 (Barahona Jiménez, 1976, pp. 359-367). Es posible que la separación de la FEUCR de la CIE -principal organización con la cual tenían relaciones internacionales- en 1968, a causa del escándalo relacionado al financiamiento por parte de la CIA a esta organización estudiantil (Altbach, 1970b, pp. 167-168; Barahona Jiménez, 1976, p. 359; "FEUCR rompe con CIE”, 1968), haya motivado reducciones en el presupuesto en este rubro.

Tabla 4

Evolución de gastos FEUCR por concepto de relaciones internacionales (1961-1968)

\begin{tabular}{|l|l|l|l|l|}
\hline Año & $\begin{array}{l}\text { Colones } \\
\text { corrientes }\end{array}$ & $\begin{array}{l}\text { Tipo de } \\
\text { cambio }\end{array}$ & $\begin{array}{l}\text { Dólares } \\
\text { corrientes }\end{array}$ & $\begin{array}{l}\text { Dólares de } \\
\mathbf{1 9 7 0}\end{array}$ \\
\hline $\mathbf{1 9 6 1}$ & 3327,25 & 5,61 & 593,09 & 769,87 \\
$\mathbf{1 9 6 2}$ & 3576,35 & 6,625 & 539,83 & 693,11 \\
$\mathbf{1 9 6 4}$ & 1399,10 & 6,625 & 211,18 & 264,50 \\
$\mathbf{1 9 6 8}$ & 1000,00 & 6,625 & 150,94 & 168,48 \\
\hline
\end{tabular}

Fuente: Balance por sumas al 31 de diciembre de 1961; Liquidación del presupuesto (al 30/09/1962); Estado de ingresos y egresos (al 31 de mayo de 1968).

Los datos relacionados a las relaciones públicas nacionales tienen las mismas problemáticas que las estadísticas ligadas a las relaciones internacionales: no fue posible obtener el dato para todos los años y en algunos casos se tratan de datos fragmentarios que no abarcan el gasto total del periodo. A raíz de los datos de la Tabla 5 es posible inferir que los gastos en este rubro se mantuvieron constantes durante la primera mitad de la década de 1960 aunque es probable que los gastos se mantuvieran o aumentaran durante el segundo quinquenio debido al enfriamiento económico de las relaciones internacionales de la Federación y el considerable aumento de los ingresos por concepto del timbre estudiantil.

\section{(c) (i) (2)}

La Revista Estudios es editada por la Universidad de Costa Rica y se distribuye bajo una Licencia Creative Commons Atribución-NoComercial-CompartirIgual 3.0 Costa Rica. Para más información envíe un mensaje a 
Tabla 5

Evolución de gastos FEUCR por concepto de relaciones públicas

$(1961-1967)^{*}$

\begin{tabular}{lllll}
\hline Año & $\begin{array}{l}\text { Colones } \\
\text { corrientes }\end{array}$ & $\begin{array}{l}\text { Tipo de } \\
\text { cambio }\end{array}$ & $\begin{array}{l}\text { Dólares } \\
\text { corrientes }\end{array}$ & Dólares de 1970 \\
\hline 1961 & $3.946,05$ & 5,61 & 703,40 & 913,06 \\
1962 & $4.285,00$ & 6,625 & 646,79 & 830,45 \\
1964 & 862,70 & 6,625 & 130,22 & 163,09 \\
1966 & $3.834,70$ & 6,625 & 578,82 & 692,73 \\
1967 & 270,00 & 6,625 & 40,75 & 47,40 \\
\hline
\end{tabular}

Fuente: Balance por sumas al 31 de diciembre de 1961; Liquidación del presupuesto (al 30/09/1962); Informe de Secretaría de Finanzas (mayo-junio 1964); Secretaría de Finanzas (08/08/1966); Estado de ingresos y egresos (al 30/06/1967).

\section{Conclusiones}

Hacia 1970, el estudiantado universitario costarricense se encontraba atravesando importantes transformaciones en su cultura política, que los impulsó a emprender varias manifestaciones y huelgas contra la transnacional ALCOA y su intento de explotar bauxita en el Pacífico Sur costarricense. Entre las principales innovaciones estudiantiles, que iban desde la participación de marchas por cuestiones no exclusivamente estudiantiles hasta arrojarle piedras al edificio de la Asamblea Legislativa, vale la pena mencionar la publicación de campos pagados en la prensa nacional. La FEUCR en ese momento administró un fondo económico destinado a publicar 10 campos en diferentes medios de comunicación de cobertura nacional (Chaves Zamora, 2016). Esos campos pagados no eran para nada baratos y no cualquier organización estaba en capacidad para hacerle frente. No obstante, a lo largo del decenio de 1960, las finanzas de la Federación tendieron a la alza facilitando la inserción de la organización en la opinión pública. La estructura de ingresos, pero especialmente el impuesto estudiantil ligado al aumento de las matrículas universitarias, permitieron que la FEUCR contara con suficientes recursos económicos para hacerle frente a la organización de elecciones estudiantiles, la elección de la Reina Universitaria, el pago de suscripción y

\section{(c) (i) ()}

La Revista Estudios es editada por la Universidad de Costa Rica y se distribuye bajo una Licencia Creative Commons Atribución-NoComercial-CompartirIgual 3.0 Costa Rica. Para más información envíe un mensaje a 

correspondencia con las organizaciones estudiantiles internacionales, el pago de salarios, el financiamiento de expediciones a comunidades indígenas, ${ }^{\text {ix }}$ la organización de congresos estudiantiles de forma regular y la gestión mediática de las protestas de 1970.

La documentación financiera de la FEUCR durante la década de 1960, además de los informes estudiados en este trabajo, incluyen informes financieros del órgano "El Universitario", facturas sueltas, inventarios de activos, comprobantes de cheques y correspondencia con instancias universitarias y extrauniversitarias. Este trabajo es apenas un acercamiento a un fenómeno poco explorado en los estudios sobre los movimientos estudiantiles. Es probable que en un futuro se pueda profundizar el análisis de los estados financieros expuestos acá debido a que la principal problemática con la que nos encontramos fue que mucha de esa documentación hace referencia a periodos de tiempo cortos y no a períodos completos de gestión. Además, el análisis de las finanzas de la FEUCR es apenas parcial porque las fuentes hacen referencia a los estados de cuenta del Consejo Superior, no a los movimientos de las respectivas asociaciones estudiantiles.

Lamentablemente, los Fondos consultados no son ricos en información sobre las finanzas de estas últimas, aunque en un futuro se podrían realizar algunos acercamientos a partir de datos parciales. Se hace necesario estudiar más a fondo el financiamiento de la organización y su relación con las políticas llevadas a cabo por la Federación y extender el estudio hacia las décadas de 1970 y 1980, con el objeto de dimensionar el impacto de la apertura de más universidades públicas y de la crisis económica en las finanzas de las organizaciones estudiantiles. 


\section{Referencias bibliográficas}

Acevedo Tarazona, A., y Samacá Alonso, G. (2011). El movimiento estudiantil colombiano como objeto de estudio en la historiografía colombiana y continental: notas para un balance y una agenda de investigación. Revista Historia y Memoria 3, 45-78.

Altbach, P. G. (1970a). The Student Internationals. An Analysis of International and Regional Student Organizations (Document Resume 24). Wisconsin: University of Wisconsin.

(1970b). The International Student Movement. Journal of Contemporary History 5(1), 156-74.

Argueta Hernández, R. A. (2004). La Asociación General de Estudiantes Universitarios Salvadoreños (AGEUS) a través de la prensa escrita (19271961) (Tesis de Maestría). Universidad de Costa Rica, Ciudad Universitaria Rodrigo Facio.

Arias Sánchez, O. (1970). Significado del movimiento estudiantil en Costa Rica. Ciudad Universitaria Rodrigo Facio: Publicaciones de la Universidad de Costa Rica.

Barahona Jiménez, L. (1972). Juventud y política. San José: Ministerio de Cultura, Juventud y Deportes.

(1976). La Universidad de Costa Rica (1940-1973). San José: Editorial de la Universidad de Costa Rica.

Bonavena, P., y Millán, M. (Eds.). (2018). Los '68 latinoamericanos. Movimientos estudiantiles, política y cultura en México, Brasil, Uruguay, Chile, Argentina y Colombia. Ciudad Autónoma de Buenos Aires: CLACSO.

Chaves Zamora, R. (09 de octubre de 2016). Estudiantes contra ALCOA en 1970: pagar para protestar. La Nación (Suplemento Áncora). Recuperado de

La Revista Estudios es editada por la Universidad de Costa Rica y se distribuye bajo una Licencia Creative Commons Atribución-NoComercial-CompartirIgual 3.0 Costa Rica. Para más información envíe un mensaje a revistaestudios.eeg@ucr.ac.cr. 
https://www.nacion.com/viva/cultura/estudiantes-contra-alcoa-en-1970-pagarpara-protestar/HM3ZPX3A3VEX7EIIX7LBAYQ2DE/story/

(2018). De estudiantes a comunistas: las manifestaciones juveniles contra ALCOA en 1970. En I. Molina Jiménez \& D. Díaz Arias (Eds.), La inolvidable edad. Jóvenes en la Costa Rica del siglo XX (pp. 103-133). Heredia: EUNA.

(2018). Fuimos jóvenes: historia y memoria de las manifestaciones estudiantiles contra Alcoa en Costa Rica, (1968-2018) (Tesis de Maestría). Universidad de Costa Rica, Ciudad Universitaria Rodrigo Facio.

(2020). Divina juventud: la Iglesia Católica y las juventudes en América Latina y Costa Rica durante la Guerra Fría (1965-1989). Revista de Historia 82, $11-$ 59.

Chávez, J. M. (2014). Catholic Action, the Second Vatican Council, and the Emergence of the New Left in El Salvador (1950-1975). The Americas (70)3, 459-487. https://doi.org/10.1017/s0003161500003990.

FEUCR rompe con CIE. (Marzo de 1968). El Universitario, pp. 1, 6.

Fonseca Zúñiga, E. (2020). Las humanidades en la génesis de la regionalización universitaria: los Estudios Generales y el origen del proceso de regionalización de la Universidad de Costa Rica. Revista Estudios (Especial: Profesores de Estudios Generales investigan). DOI 10.15517/re.v0i0.40925

Fumero, P. (1997). 'Se trata de una dictadura sui generis'. La Universidad de Costa Rica y la Guerra Civil de 1948. Anuario de Estudios Centroamericanos 23(1-2), $115-42$.

González Ortega, A. (2005). Mujeres y hombres de la posguerra costarricense (1950-1960). San José: Editorial de la Universidad de Costa Rica.

González Villalobos, P. (1987). Los orígenes del movimiento estudiantil universitario en Costa Rica (1844-1940) (Avances de Investigación 38). San José: Centro

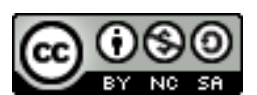

La Revista Estudios es editada por la Universidad de Costa Rica y se distribuye bajo una Licencia Creative Commons Atribución-NoComercial-CompartirIgual 3.0 Costa Rica. Para más información envíe un mensaje a revistaestudios.eeg@ucr.ac.cr. 
de Investigaciones Históricas.

González, P. (1985). Las luchas estudiantiles en Centroamérica 1970-1983. En D. Camacho y R. Menjívar (Coords.), Movimientos populares en Centroamérica (238-292). San José: EDUCA.

Gutiérrez Slon, J. A. (2015). Mundos juveniles en movimientos estudiantiles: historia, vida cotidiana y acciones de lucha en la FEUNA, 1973-2012 (Tesis de Maestría), Universidad de Costa Rica, Ciudad Universitaria Rodrigo Facio.

Gutiérrez, C. J. (1969). Student Participation in the Government of the University of Costa Rica. The American Journal of Comparative Law 17(3), 390-394.

Historia de la FEUCR. (Junio de 1967). El Universitario, p. 7.

Hobsbawm, E. (1999). Historia del siglo XX. Buenos Aires: Crítica.

Klimke, M., y Nolan, M. (2018). Introduction: the Globalization of the Sixties. En C. Jian, M. Klimke, M. Kirasirova, M. Young \& J. Waley-Cohen (Eds.), The Routledge Handbook of the Global Sixties: Between Protest and Nation Building (pp. 1-9). New York: Routledge.

Kruijt, D. (2009). Guerrillas: guerra y paz en Centroamérica. Guatemala: F\&G Editores.

Maanen, G. V. (1966). The International Student Movement. History and Background. The Hague: International Documentation and Information Centre.

Molina Jiménez, I. (2009). Anticomunismo reformista, competencia electoral y cuestión social en Costa Rica 1931-1948. San José: Editorial Costa Rica. (2016). La educación en Costa Rica. De la época colonial al presente. San José: EDUPUC.

(2017). Estadísticas de financiamiento, salarios docentes, matrícula, cobertura y graduación en la educación costarricense: una contribución

\section{(c) (i) (2)}

La Revista Estudios es editada por la Universidad de Costa Rica y se distribuye bajo una Licencia Creative Commons Atribución-NoComercial-CompartirIgual 3.0 Costa Rica. Para más información envíe un mensaje a revistaestudios.eeg@ucr.ac.cr. 

documental (1827-2017) (Cuadernos del Bicentenario 1). San José: Centro de Investigaciones Históricas de América Central.

(2018). El financiamiento educativo público en Costa Rica a largo plazo (1860-2016). Historia y Memoria 16, 165-98. https://doi.org/10.19053/20275137.n16.2018.5972.

(2019a). Huelgas democratizadoras. La rebelión estudiantil en el Instituto Tecnológico de Costa Rica (1980-1982). San José: CIHAC, EDUPUC. (2019b). La primera marcha por presupuesto universitario en Costa Rica. Del Pasado y del Presente 1(1). Recuperado de https://www.academia.edu/41329759/La primera marcha por presupuesto universitario en Costa Rica (2020). Los conflictos por la política de admisión de la Universidad de Costa Rica durante la década de 1950. Historia y Memoria de la Educación 11, 281315. https://doi.org/10.5944/hme.11.2020.22839.

Molina Jiménez, I. y Díaz Arias, D. (2017). Epílogo: un inmenso y generoso corazón. En I. Molina Jiménez y D. Díaz Arias (Eds.), El verdadero anticomunismo. Política, género y Guerra Fría en Costa Rica (1948-1973) (pp. 285-95). San José: EUNED.

Molina Jiménez, I. y Díaz Arias, D. (Eds.). (2018). La inolvidable edad. Jóvenes en la Costa Rica del siglo XX. Heredia: EUNA.

Picado Rojas, C. A. (1991). Las políticas estatales de juventud: el caso de Costa Rica (1980-1989) (Tesis de Licenciatura). Universidad de Costa Rica, Ciudad Universitaria Rodrigo Facio.

Pozzi, P. A. y Pérez, C. (Eds.). (2012). Por el camino del Che. Las guerrillas latinoamericanas 1959-1990. Buenos Aires: Imago Mundi.

Salazar Montes, M. (2018). Rebelión juvenil y régimen político (1962-1971). En I.

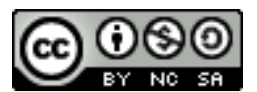

La Revista Estudios es editada por la Universidad de Costa Rica y se distribuye bajo una Licencia Creative Commons Atribución-NoComercial-CompartirIgual 3.0 Costa Rica. Para más información envíe un mensaje a revistaestudios.eeg@ucr.ac.cr. 
Revista Estudios, (41), 2020.

Diciembre 2020-Mayo 2021

ISSN 1659-3316

Garita Mondragón Marco

Molina Jiménez \& D. Díaz Arias (Eds.), La inolvidable edad. Jóvenes en la Costa Rica del siglo XX, (pp. 81-101). Heredia: EUNA.

Salazar Montes, M. F. (2013). Los espectáculos de representación escénico-popular en Costa Rica: culturas populares y políticas culturales, durante 1960-1990 (Tesis de Maestría). Universidad de Costa Rica, Ciudad Universitaria Rodrigo Facio.

Souto Kustrín, S. (2007). Juventud, teoría e historia: la formación de un sujeto social y de un objeto de análisis. Historia Actual Online 13, 171-92.

Torres-Rivas, E., Bronfenmajer, G., Cassá, R., Molina Chocano, G., y James-Bryan, M. (1989). Escépticos, narcisos, rebeldes: 6 estudios sobre la juventud. San José: FLACSO.

Universidad de Costa Rica. (1955). Anales de la Universidad de Costa Rica 1954. San José. (1957). Anales de la Universidad de Costa Rica 1956. San José. (1958). Anales de la Universidad de Costa Rica 1957. San José: Hnos. Saborío Esquivel LTDA.

\section{Notas}

\footnotetext{
${ }^{\mathrm{i}}$ Agradezco a las y los funcionarios de dicha institución la disposición y ayuda que me brindaron en la búsqueda de fuentes primarias, especialmente la ayuda brindada por Ivannia Azofeifa Rojas.

ii AUROL, Fondo FEUCR, Caja 4, Libro de Actas, Acta N`37 del Consejo Superior (07/01/1960), f. 184-185.

iii AUROL, Fondo FEUCR, Caja 4, Correspondencia enviada y recibida, Sesión CU N 1059 (14/03/1960).

${ }^{\text {iv }}$ AUROL, Fondo FEUCR, Caja 5, Correspondencia enviada y recibida, Balance por sumas al 31 de diciembre de 1961.

${ }^{v}$ AUROL, Fondo FEUCR, Caja 5, Correspondencia enviada y recibida, Estado de ingresos y egresos al 30 de setiembre de 1962.

vi AUROL, Fondo FEUCR, Caja 12, Correspondencia enviada y recibida, Artículos importantes del Reglamento electoral (1970).

vii AUROL, Fondo FEUCR, Caja 12, Correspondencia enviada y recibida, Recibí de la A.E.I. (24/06/1970).

viii AUROL, Fondo FEUCR, Caja 11, Correspondencia enviada y recibida, Inscripción patronal (02/01/1968).

${ }^{\text {ix }}$ AUROL, Fondo FEUCR, Caja 11, Informe poblaciones zona sur (1968).
}

\section{(c) (i) (2)}

La Revista Estudios es editada por la Universidad de Costa Rica y se distribuye bajo una Licencia Creative Commons Atribución-NoComercial-CompartirIgual 3.0 Costa Rica. Para más información envíe un mensaje a revistaestudios.eeg@ucr.ac.cr. 